\title{
Ethylene signaling involves in seeds germination upon submergence and antioxidant response elicited confers submergence tolerance to rice seedlings
}

\author{
Yi-Chun Huang, Tsun-Hao Yeh and Chin-Ying Yang ${ }^{*}$ (i)
}

\begin{abstract}
Background: Flooding has negative impact on agriculture. The plant hormone ethylene is involved in plant growth and stress responses, which are important role in tolerance and adaptation regulatory mechanisms during submergence stress. Ethylene signaling crosstalk with gibberellin signaling enhances tolerance in lowland rice (Flood Resistant 13A) through a quiescence strategy or in deepwater rice through an escape strategy when rice is submerged. Information regarding ethylene-mediated priming in submergence stress tolerance in rice is scant. Here, we used 1-aminocyclopropane-1-carboxylic acid, an ethylene precursor, to evaluate the response in submerged rice seedlings.

Results: The germination rate and mean germination times of rice seeds was higher in seedlings under submergence only when ethylene signaling was inhibited by supplemented with silver nitrate $\left(\mathrm{AgNO}_{3}\right)$. Reduced leaf chlorophyll contents and induced senescence-associated genes in rice seedlings under submergence were relieved by pretreatment with an ethylene precursor. The ethylene-mediated priming by pretreatment with an ethylene precursor enhanced the survival rate and hydrogen peroxide $\left(\mathrm{H}_{2} \mathrm{O}_{2}\right)$ and superoxide $\left(\mathrm{O}_{2}{ }^{-}\right)$anion accumulation and affected antioxidant response in rice seedlings.

Conclusions: Pretreatment with an ethylene precursor leads to reactive oxygen species generation, which in turn triggered the antioxidant response system, thus improving the tolerance of rice seedlings to complete submergence stress. Thus, $\mathrm{H}_{2} \mathrm{O}_{2}$ signaling may contribute to ethylene-mediated priming to submergence stress tolerance in rice seedlings.
\end{abstract}

Keywords: Rice, Submergence, Ethylene, Reactive oxygen species, Antioxidant enzyme activity

\section{Background}

Severe climate-related disasters include flooding due to increased frequency of heavy rain. Flooding affects agriculture causing outright crop yield losses. The term flooding comprises both waterlogging and submergence. The pore space in the soil is filled with water when soil is under excess water stress; this decreases soil oxygen levels, limits gas diffusion and soil nutrient effusion, and impairs plant growth and development (Nishiuchi et al. 2012). With increased submergence duration, tiller

\footnotetext{
* Correspondence: emiyang@dragon.nchu.edu.tw

Department of Agronomy, National Chung Hsing University, Taichung 40227, Taiwan
}

number, green leaves number, and dry weight of rice decreases. The survival and growth of rice are severely affected by submergence (Reddy and Mittra 1985; Gautam et al. 2017; Wu and Yang 2016).

Unfavorable conditions activate phytohormonal signals in plants, in turn enhancing their tolerance to environmental stress. The gaseous plant hormone ethylene mediated developmental processes and stress tolerance, such as seed germination, senescence, and stress responses ( $\mathrm{Yu}$ et al. 2017; Xia et al. 2015; El-Maarouf-Bouteau et al. 2015). Ethylene signalinginduced mitogen-activated protein kinase (MAPK) cascades can be activated with the application of the 
ethylene precursor 1-aminocyclopropane-1-carboxylic acid (ACC) in Medicago and Arabidopsis (Ouaked et al. 2003). Under salt stress, ethylene can activate the MAPK cascade and enhance reactive oxygen species (ROS) generation (Teige et al. 2004). The interplay of ethylene signaling and ROS production activates the antioxidant defense system for flooding responses in rice (Steffens 2014; Yang and Hong 2015).

Several studies have reported that ethylene is crucial against hypoxia signal-inducing flooding stress. Aerenchyma formation can be induced in maize roots by applying ethylene in flooded conditions (Rajhi et al. 2011). Ethylene signaling triggers the process of programmed cell death resulting in ethylene-responsive lysigenous aerenchyma formation (Guo et al. 2015; Muhlenbock et al. 2007; Chen et al. 2002). Two key ethylene biosynthesis enzymes, 1-aminocyclopropane-1-carboxylic acid synthase (ACS) and 1-aminocyclopropane-1-carboxylic acid oxidase (ACO), are involved in plant response to hypoxia stress. ACS converts S-adenosylmethionine (AdoMet) into ACC and the byproduct $5^{\prime}$-methylthioadenosine; then, ACO converts ACC to ethylene, thus increasing the ethylene levels (Rzewuski and Sauter 2008; Adams and Yang 1979). In this study, to further clarify the role of ethylene signaling during submergence, we used ACC, an ethylene precursor, to evaluate the response in submerged rice seedlings.

\section{Results}

\section{Submergence-induced germination inhibition was} alleviated after ethylene signaling was blocked

To evaluate the effect of ethylene signaling on rice seed germination during submergence, we pretreated rice seeds under normoxia (Nor), submergence (Sub), and submergence supplemented with silver nitrate $\left(\mathrm{Sub}+\mathrm{AgNO}_{3}\right)$ for 2 days, and then, we again placed the seeds under normal condition to calculate the seed germination rates and mean germination times (MGTs). $\mathrm{Ag}^{+2}$ ions in $\mathrm{AgNO}_{3}$ inhibit the action of ethylene by reducing the ethylene receptor's capacity to bind ethylene (Yang 1985; Kumar et al. 2009). Our results presented that the seeds pretreated under submergence germinated slower than those under Nor. However, germination inhibition was alleviated under submergence only when supplemented with $\mathrm{AgNO}_{3}$ (Fig. 1a). MGT of seeds pretreated under Nor, Sub, and $\mathrm{Sub}+\mathrm{AgNO}_{3}$ was 1.94, 2.87, and 2.66 days, respectively (Fig. 1b). Thus, submergence combined with the ethylene signaling inhibition reduced MGT.

\section{Survival rate was enhanced after pretreatment with ethylene precursor in rice seedlings subjected to submergence stress}

Ethylene, involved in hypoxia signaling, affects anaerobic gene expression and ethanolic fermentation in plants (Yang et al. 2011; Peng et al. 2001). To investigate the

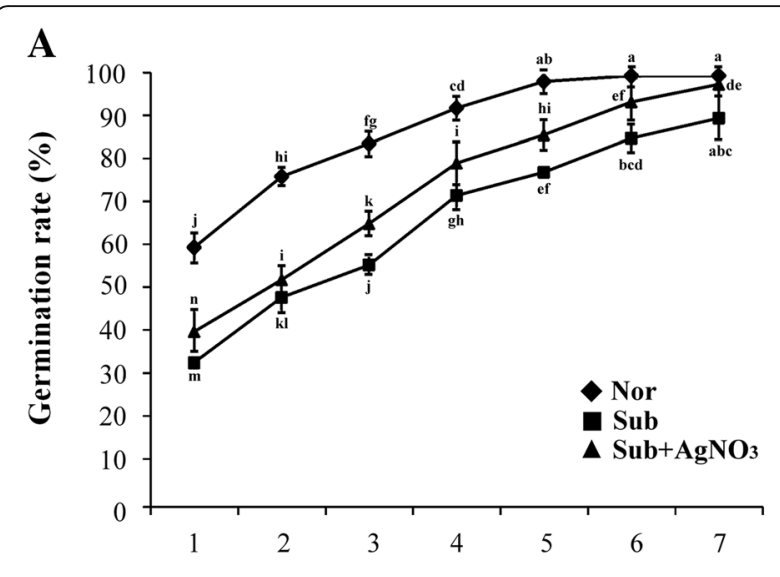

Days after submergence

\section{B}

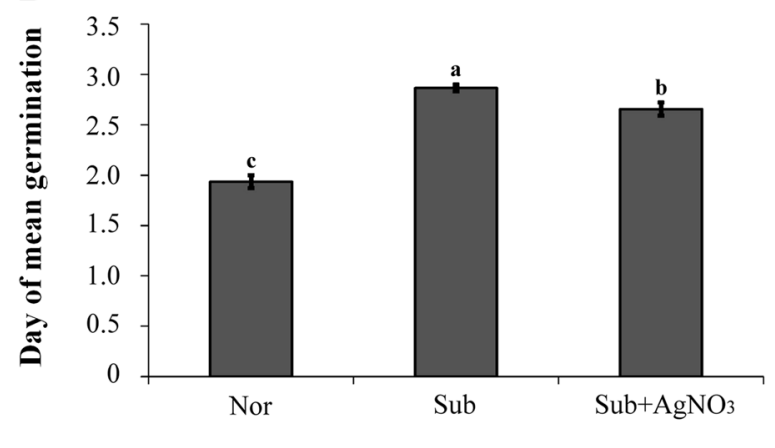

Treatments

Fig. 1 The germination assay of rice seeds under submergence combined with inhibition of ethylene signaling conditions. Seeds were surface sterilized and placed on a wet filter paper under Nor, $\mathrm{Sub}$, and $\mathrm{Sub}+\mathrm{AgNO}_{3}$ for pretreatment for 2 days and subsequently placed under Nor in a growth chamber for the germination assay. Seed germination was observed for 1, 2, 3, 4, 5, 6, and 7 days. a The germination rate of TK9 rice seeds. $\mathbf{b}$ The day of mean germination. The data represent the average values \pm SD from 30 seedlings of each treatment obtained from three biologically independent experiments. The values with different letters are significantly different at $P<0.05$, according to one-way ANOVA with post hoc Duncan's test

effects of ethylene-mediated priming on submergence stress tolerance, the 8-day-old Tai-keng 9 (TK9) rice seedlings were grown on Kimura B medium with or without ACC pretreatment for 2 days; then, they were subjected to complete submergence for $4,6,8$, and 10 days and finally allowed to recover for 10 days. The survival rate was assessed on the basis of ability to form one or more new leaves. ACC is an effective precursor of ethylene in higher plants. The survival rates of TK9 rice seedlings without and with ACC pretreatment followed by complete submergence for $4,6,8$, and 10 days were $92.5 \%, 76.3 \%, 50.0 \%$, and $18.8 \%$, respectively, and $95.0 \%, 80.0 \%, 62.5 \%$, and $37.8 \%$, respectively (Fig. $2 \mathrm{a}$ and b). Thus, survival rates significantly increased after 


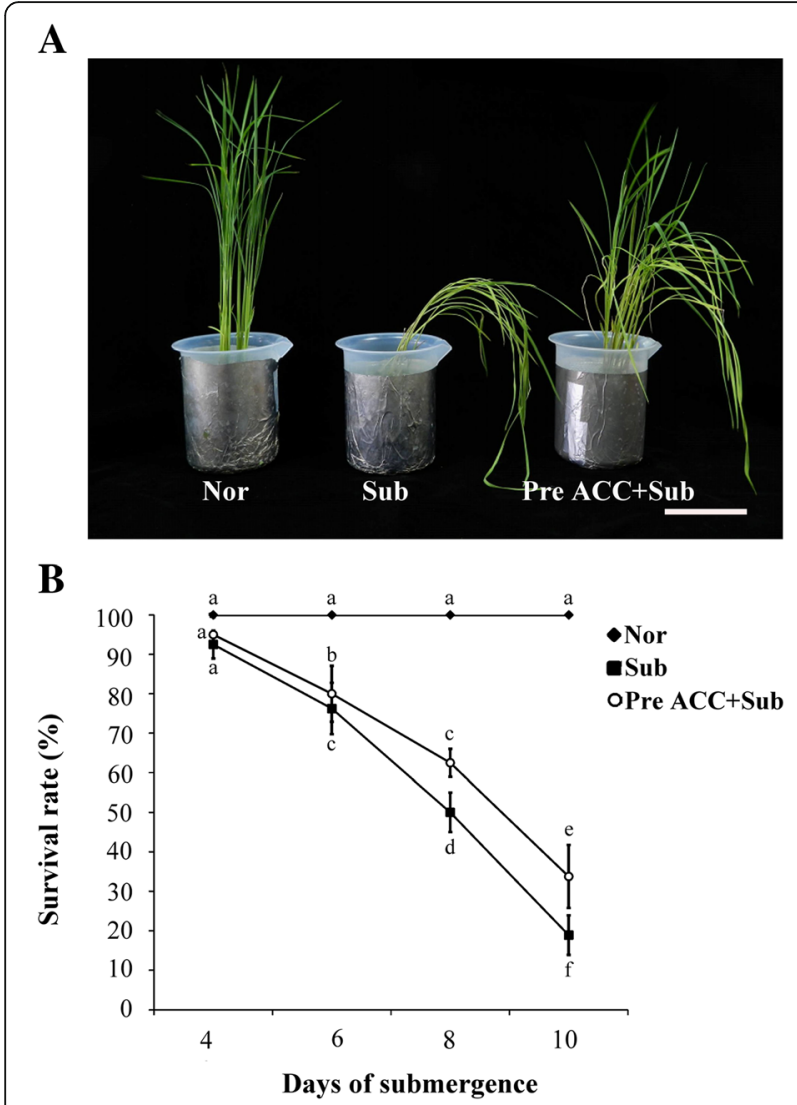

Fig. 2 Tolerance determination of rice seedlings under submergence by pretreatment with an ethylene precursor. a The phenotypes of 8-day-old TK9 rice seedlings treated under Nor, Sub, and Pre ACC + Sub for 2 days and then subjected to submergence for 10 days and subsequent recovery for 10 days. $B a r=1 \mathrm{~cm}$. The photograph exhibits the results in three independent seedlings. $\mathbf{b}$ The survival rate of TK9 rice seedlings treated under Nor, Sub, and Pre ACC + Sub for 2 days and then subjected to submergence for $4,6,8$, and 10 days followed by 10-day recovery. The data represent the average values \pm SD from 30 seedlings of each treatment obtained from three biologically independent experiments. The values with different letters are significantly different at $P<0.05$, according to one-way ANOVA with post hoc Duncan's test

pretreatment with an ethylene precursor in TK9 seedlings under submergence stress.

\section{Reduced leaf chlorophyll contents and induced senescence-associated genes in rice seedlings under submergence were alleviated after ethylene precursor pretreatment}

The chlorophyll contents and senescence-assocaited genes (SAGs) are severely affected under complete submergence (Wu and Yang 2016). To understand the effects of ethylene-mediated priming in chlorophyll contents during submergence, the 8-day-old TK9 rice seedlings were pretreated with or without ACC for 2 days and then subjected to complete submergence for 6,8 , and 10 days to measure chlorophyll contents. Seedlings pretreated ACC with under complete submergence demonstrated higher chlorophyll $\mathrm{b}$ and total chlorophyll content than did untreated seedlings under submergence (Fig. 3).

The mRNA expression of the SAGs red chlorophyll catabolite reductase 1 (RCCR1; involved in chlorophyll degradation) (Pruzinska et al. 2007), and isocitrate lyase (Osl85; highly induced by prolonged darkness and natural senescence) (Yamada et al. 2014) was determine through quantitative reverse transcription polymerase chain reaction (qRT-PCR). The results indicated that the induction of RCCR1 and Osl85 expression was significantly lower in seedlings under complete submergence with ACC pretreatment than in those under submergence only (Fig. 4). We also had detected some genes expression that involved in chlorophyll biosynthesis and degradation such as CAO1, HEMA1, NYC1 and NOL by qRT-PCR. The results presented no significant different in our experiment treatment (Additional file 1: Figure S1). Thus, pretreatment of rice seedlings with an ethylene
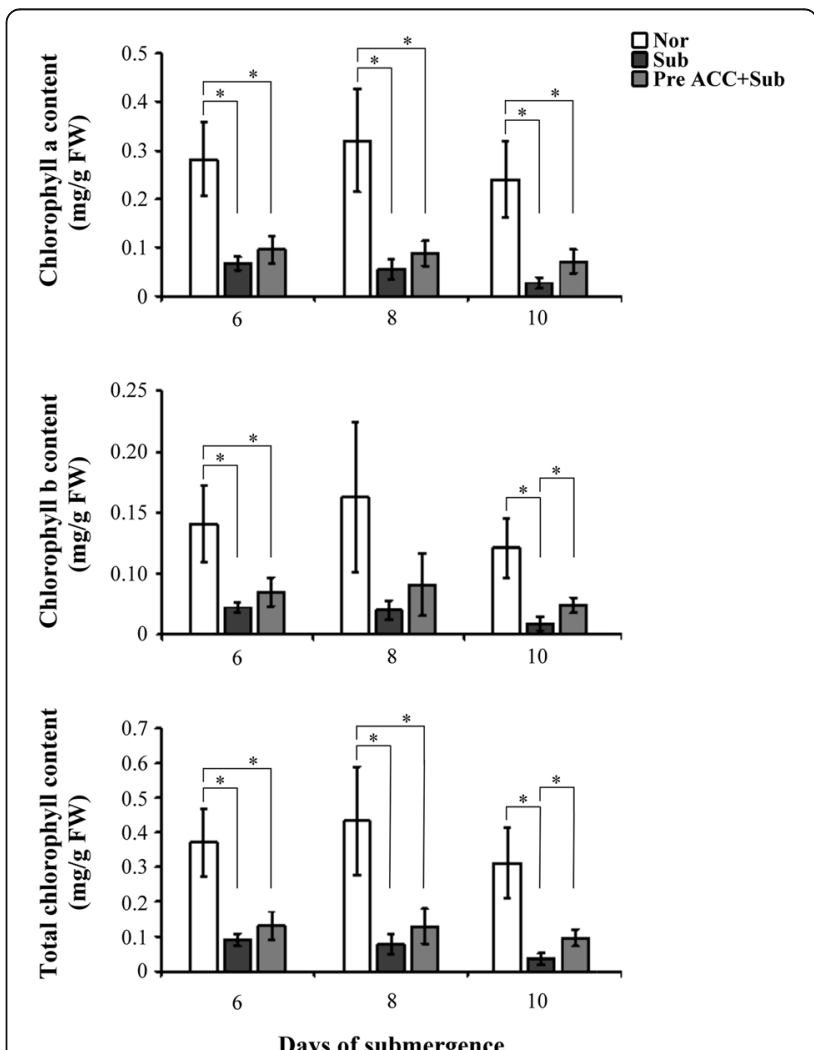

Fig. 3 The chlorophyll content of rice seedlings by pretreatment with an ethylene precursor under submergence conditions. The chlorophyll a, b and total content of 8-day-old TK9 rice seedlings treated under Nor, Sub, and Pre ACC + Sub for 2 days and then subjected to submergence for $4,6,8$, and 10 days. The data represent the average values \pm SD from four biologically independent experiments. The values with asterisk are significantly different at $P<0.05$, according to Student's $t$ test 


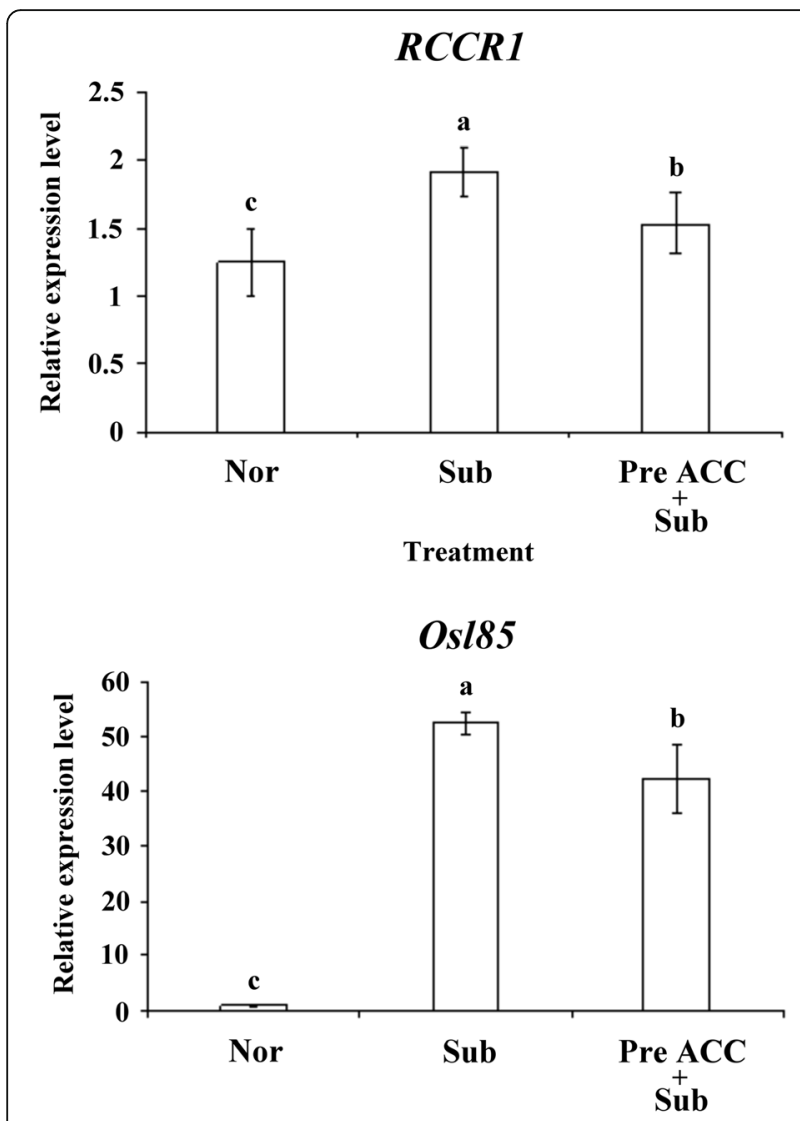

Treatment

Fig. 4 The transcript levels of SAGs in rice seedlings by pretreatment with an ethylene precursor under submergence conditions. qRT-PCR quantified the transcript levels of RCCR1 and Os/85 genes in 8-dayold TK9 rice seedlings treated under Nor, Sub, and Pre ACC + Sub for 2 days and then subjected to submergence for 4 days. The data represent the average values \pm SD from three biologically independent experiments. The values with different letters are significantly different at $P<0.05$, according to one-way ANOVA with post hoc Duncan's test

precursor significantly affected the reduction of leaf chlorophyll content and induction of SAG expression under submergence stress.

\section{Pretreatment with an ethylene precursor lead to} hydrogen peroxide and superoxide ion accumulation and affected antioxidant response during submergence

ROS is involved in ethylene-dependent and -independent submergence adaptation (Yang and Hong 2015). To determine how ethylene-mediated priming affects antioxidant system homeostasis, ROS accumulation and antioxidant enzyme activity were detected. The 8-day-old TK9 rice seedlings were pretreated with and without ACC for 2 days and then subjected to complete submergence for 4 days; superoxide ion $\left(\mathrm{O}_{2}{ }^{-}\right)$and hydrogen peroxide $\left(\mathrm{H}_{2} \mathrm{O}_{2}\right)$ accumulation was detected by staining with nitro blue tetrazolium (NBT) and 3,3'-diaminobenzidine (DAB). The staining of first leaf revealed higher $\mathrm{O}_{2}{ }^{-}$and $\mathrm{H}_{2} \mathrm{O}_{2}$ accumulation under complete submergence after pretreatment with ACC than under submergence alone (Fig. 5a).

The activities of antioxidative enzymes, namely catalase (CAT), ascorbate peroxidase (APX), superoxide dismutase (SOD), and total peroxidase (POX), were then determined after complete submergence with or without ACC pretreatment. The CAT, SOD, and APX activities decreased under submergence stress with or without ACC pretreatment. However, SOD activity significantly decreased after ACC pretreatment under submergence. POX activity increased under submergence stress; it was particularly higher under submergence after ACC pretreatment (Fig. 5b). Thus, pretreatment with an ethylene precursor may affect intracellular redox homeostasis and antioxidant systems under submergence stress.

\section{Discussion}

The plant hormone ethylene plays important roles in plant adaptation to submergence stress. It is the principal factor initiating fast underwater elongation of leaves or stems-the so-called escape strategy in deepwater rice (Hattori et al. 2011). In lowland rice Flood Resistant $13 \mathrm{~A}$, it is elicits a quiescence strategy based on suppression of elongation to avoid energy consumption during flash flooding (Manzur et al. 2009). Studies presented that some QTLs (quantitative trait loci) associated with tolerance of flooding during germination have been identified that revealed $\mathrm{ABA}$ and $\mathrm{GA}$ involved in submergence tolerance during germination (Miro and Ismail, 2013). The role of ethylene in priming during submergence stress remains ambiguous. To further understand the effects of ethylene-mediated priming on tolerance and antioxidant response to submergence stress, we used an ethylene precursor ACC to investigate change in physiological and molecular responses in rice seedlings under submergence stress. Studies have revealed the roles of ethylene in the release of primary and secondary dormancy and the germination of nondormant seeds under normal and stressed conditions in many plant species (Petruzzelli et al. 2000; Kepczynski and Kepczynska 1997); poor germination is a feature of the Arabidopsis ethylene-insensitive mutant (Johnson and Ecker 1998). Waterlogging or submergence causes a rapid decline in dissolved oxygen concentrations in the soil water, thus resulting in seed germination failure and lengthening the germination time in pea, oak, and lupin seeds (Sarlistyaningsih et al. 1995; Perez-Ramos and Maranon 2009; Jackson and Hall 1987). In the current study, the germination rate of TK9 rice seeds considerably decreased but their MGT considerably increased under the Sub condition compare with that under the Nor condition (Fig.1 a and b). However, germination 
A

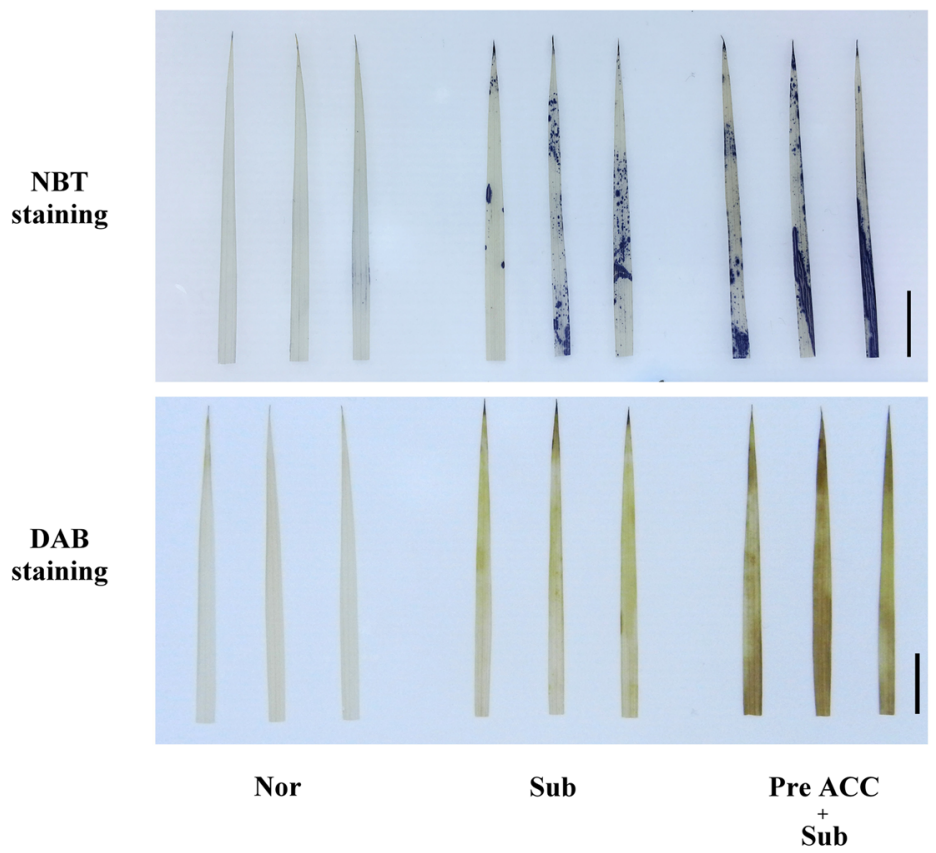

B
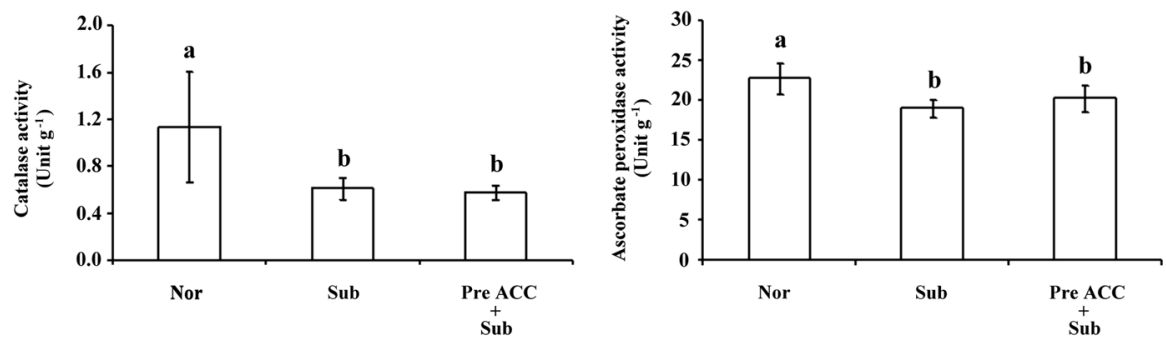

Treatment

Treatment
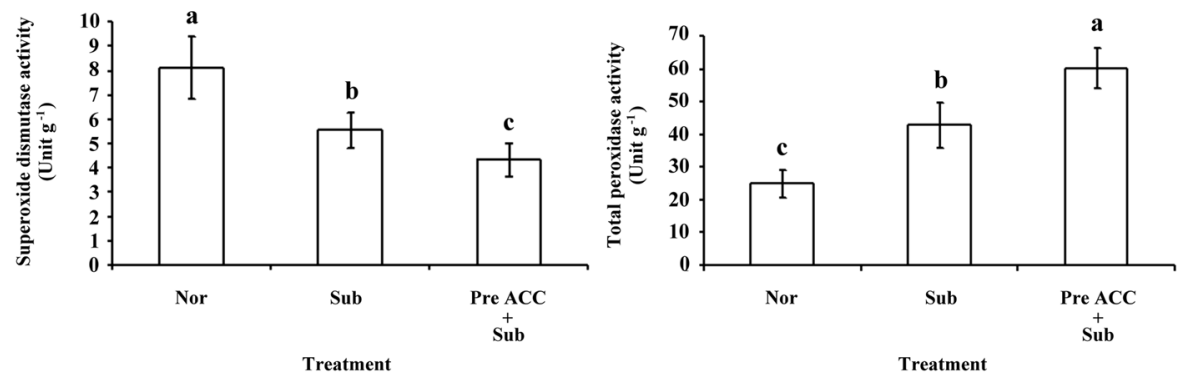

Fig. 5 The detection of $\mathrm{O}_{2}{ }^{-}$and $\mathrm{H}_{2} \mathrm{O}_{2}$ accumulation and antioxidative enzyme activity assay in rice seedlings pretreated with an ethylene precursor under submergence conditions. a The NBT and DAB staining methods for detecting the accumulation of $\mathrm{O}_{2}{ }^{-}$and $\mathrm{H}_{2} \mathrm{O}_{2}$ in detached leaves of 8-day-old TK9 rice seedlings treated under Nor, Sub, and Pre ACC + Sub for 2 days and then subjected to submergence for 4 days. Same results were obtained in three independent experiments. Photograph exhibits results of three independent leaves. Bar $=1 \mathrm{~cm}$. $\mathbf{b}$ The enzyme activity of detached leaves of 8-day-old TK9 rice seedlings treated under Nor, Sub, and Pre ACC + Sub for 2 days and then subjected to submergence for 4 days. CAT, APX, SOD, and POX activities are the average values \pm standard deviation of three biologically independent experiments each with duplicate samples. The values with different letters are significantly different at $P<0.05$, according to a one-way ANOVA with post hoc Duncan's test

inhibition was partially disrupted under submergence in $\mathrm{Sub}+\mathrm{AgNO}_{3}$-treated seedlings because ethylene signaling was inhibited through blocking ethylene perception with silver ions (Fig. $1 \mathrm{a}$ and b). Therefore, the results imply that in addition to ethylene signaling, other pathways are involved in the regulation of seed germination under submergence stress. Studies indicated that silver ions affects not only ethylene signaling may also auxin 
efflux to affect root elongation (Strander et al., 2009). Whether the auxin signaling involved in the regulation of seed germination under submergence stress need further research.

Complete submergence of rice plants can severely delay physiological responses, retard growth and development, reduce yield, and even cause death (Jackson and Ram 2003; Yang et al. 2017). We demonstrated that the survival rate of rice seedlings can be improved under complete submergence stress through ACC pretreatment (Fig. 2). Moreover, under submergence, chlorophylls $\mathrm{b}$ and total chlorophyll contents could be maintained by ACC pretreatment (Fig. 3). Under complete submergence, ACC-pretreated seedlings demonstrated lower SAG mRNA expression than did untreated seedlings (Fig. 4). Thus, ethylene-mediated priming has senescence inhibition-associated positive effects on plant submergence tolerance.

ROS plays a key role in signal transduction in cells (Mittler et al. 2011). Homeostatic regulation of ROSantioxidant interactions in plant cells confers increased to environmental stress tolerance to the plants. Cellular antioxidants influence plant growth and development by modulating processes from cell division and cell elongation to senescence and death (Foyer and Noctor 2005). In plants, complex intracellular mechanisms regulate the ROS production and scavenging, particularly under stress. In our study, $\mathrm{H}_{2} \mathrm{O}_{2}$ accumulation and POX activity increased under submergence with ACC pretreatment compared with that under submergence alone (Fig. 5). These results may imply that $\mathrm{H}_{2} \mathrm{O}_{2}$ signaling contributes to ethylene-mediated priming on submergence tolerance in rice seedlings. Taken together, this study demonstrated that ACC pretreatment trigger positive priming mechanism to increase plant tolerance to submergence.

\section{Conclusions}

In conclusion, our results demonstrated that the germination rates of rice seeds under submergence partially increased after ethylene signaling was inhibited. In rice seedlings, ethylene-mediated priming through pretreatment with an ethylene precursor modulated leaf chlorophyll content and SAG expression, enhanced survival, increased $\mathrm{H}_{2} \mathrm{O}_{2}$ and $\mathrm{O}_{2}{ }^{-}$accumulation, and reduced antioxidant response was affected by. Thus, seed germination and rice seedling tolerance can be improved under complete submergence by modulating ethylene signaling because ethylene-mediated priming affects senescence induction and ROS and antioxidant response conferring submergence tolerance to rice. Whether this regulatory mechanism can crosstalk with other pathways remains unclear and merits further study.

\section{Materials and methods}

\section{Plant materials and growth conditions}

TK9 rice (Oryza sativa Japonica) was used in this study. Rice seeds were sterilized by dipping in 3\% sodium hypochlorite solution for $30 \mathrm{~min}$, followed by gentle washing with distilled water for at least four to five times. The sterilized seeds were subsequently placed on a wet filter paper for 3 days at $28^{\circ} \mathrm{C}$ under a 16-h light8 -h dark cycle in a growth chamber. The germinated seeds were transferred onto a metal grid placed over a 500-mL beaker containing Kimura B medium for growth. For the seed germination assay, data were collected from 30 seeds for each treatment in three independent experiments. Seeds were placed under Nor, in 4.5-cm-deep water in a water tank (Sub), or in 4.5-cm-deep water containing $10 \mu \mathrm{M} \mathrm{AgNO}_{3}$ in a water tank $\left(\mathrm{Sub}+\mathrm{AgNO}_{3}\right)$ for pretreatment for 2 days; then, the seeds were transferred onto a wet filter paper for germination. Germination was confirmed when the radicles were $1 \mathrm{~mm}$ long. Germination percentage was recorded every $24 \mathrm{~h}$ for 7 days. The number of germinated seeds was expressed as a percentage of the total number of seeds plated for the indicated periods. MGTs were calculated to assess the time required for germination (Matthews and Khajeh-Hosseini 2007).

\section{Seedlings submergence, ACC treatment, and survival rate determination}

For submergence treatment, 8-day-old seedlings were completely submerged with or without $10 \mu \mathrm{M}$ ACC pretreatment for 2 days, followed by transferring into a water tank $\left(40 \times 40 \times 60 \mathrm{~cm}^{3}\right)$ with 55-cm-deep water for $4,6,8$, and 10 days under a 16 -h light-8-h dark cycle. Under Nor, seedlings were placed under normal condition for the indicated periods. The water was drained out for subsequent 10-day recovery. The ability to grow new leaves after 10-day recovery was considered the measure of survival. Experiments were repeated three times, and at least 30 seedlings were measured independently each time. After each treatment, samples tissues were immediately frozen in liquid nitrogen and stored at $-80^{\circ} \mathrm{C}$ for further assay.

\section{Plant chlorophyll content measurements and qRT-PCR analyses}

For chlorophyll content assay, the 8-day-old seedlings were treated under Nor and Sub with and without $10 \mu \mathrm{M}$ ACC pretreatment for 2 days followed by submergence for 6,8 , and 10 days. Above-ground tissue $(50 \mathrm{mg}$ ) was collected and ground in $2 \mathrm{~mL}$ of sodium phosphate buffer $(50 \mathrm{mM}, \mathrm{pH} 6.8) ; 40 \mu \mathrm{L}$ of this solution was added to $960 \mathrm{~mL}$ of $99 \%$ ethanol and incubated for $30 \mathrm{~min}$ at room temperature in the dark with gentle shaking. After centrifugation at $4{ }^{\circ} \mathrm{C}$ for $15 \mathrm{~min}$ at 1000 
$\mathrm{g}$, the absorbance of the supernatant was measured at 665 and $649 \mathrm{~nm}$ using a spectrophotometer (Metertec SP8001) for determining chlorophyll $\mathrm{a}$ and $\mathrm{b}$ and total chlorophyll contents. The values were collected from three biologically independent experiments.

For qRT-PCR analyses, total RNA was extracted using TRIzol (Invitrogen, Carlsbad, CA, USA) and then subjected to DNase treatment using the TURBO DNA-free Kit (Ambion, Austin, TX, USA). RNA concentration was determined, and samples were then reverse transcribed into cDNA by using Moloney murine leukemia virus reverse transcriptase (Invitrogen). qRT-PCR was performed as previously described (Yang et al. 2017) using a Bio-Rad CFX instrument (CFX Connect ${ }^{\mathrm{Tm}}$, Bio-Rad, USA) with Power SYBR Green PCR Master Mix (GeneMark, Taipei, Taiwan), according to the manufacturer's recommendations. The ubiquitin gene was used as an internal control for normalization. Relative expression levels were analyzed using Bio-Rad CFX Manager (version 3.1). Experiments were repeated three times independently with duplicate samples. The primer sequences for qRT-PCR are presented in Table 1.

\section{Histochemical staining and antioxidative enzyme activity assay}

The detached leaves of 8-day-old rice seedlings were treated under Nor, Sub, and submergence supplemented with $10 \mu \mathrm{M}$ ACC (Pre ACC + Sub) for 2 days and then submerged for 4 days. $\mathrm{O}_{2}{ }^{-}$and $\mathrm{H}_{2} \mathrm{O}_{2}$ accumulation in cells was observed through the NBT and DAB staining methods, as previously described (Yang and Hong 2015). The results were obtained from three independent experiments. For the antioxidative enzyme assay, shoot tissue $(50 \mathrm{mg})$ was excised and immediately used for

Table 1 Primers used for quantitative RT-PCR experiments

\begin{tabular}{|c|c|}
\hline Gene name & Primer sequence \\
\hline Os/85 - forward & $5^{\prime}$-catgggcaaaggagttactgaagag $-3^{\prime}$ \\
\hline Osl85 - reverse & 5'-ggatttggcaagaacatggctg - 3' \\
\hline OsRCCR1 - forward & $5^{\prime}$-gcaccttctcactgacagcaatac $-3^{\prime}$ \\
\hline OsRCCR1 - reverse & $5^{\prime}$-accacgcactatctcttccaagg $-3^{\prime}$ \\
\hline Osubiquitin - forward & 5'-aaccagctgaggcccaaga-3' \\
\hline Osubiquitin - reverse & 5'-acgattgatttaaccagtccatga-3' \\
\hline OsCAO1 - forward & $5^{\prime}$-tgctcatcaagccttccttcaggtg-3' \\
\hline OsCAO1 - reverse & $5^{\prime}$-ctcgactgatacgtttcttgttgcg-3' \\
\hline OSHEMA1 - forward & 5'-aggaaagaagtagcatagcg-3' \\
\hline OsHEMA1 - reverse & $5^{\prime}$-cgatagagtcttgagtggtc-3' \\
\hline OsNYC1 - forward & $5^{\prime}-$ cacactgcttctcctggaatgg-3' \\
\hline OsNYC1 - reverse & $5^{\prime}$-ctacgctcaacactttccttcacc-3' \\
\hline OsNOL - forward & 5'-ccacgaaaggtataggatatg-3' \\
\hline OsNOL - reverse & 5'-tcaagtcagtcaccgcagat-3' \\
\hline
\end{tabular}

enzyme extraction. The levels of CAT, APX, POX, and SOD activity were analyzed as previously described (Wu and Yang 2016). Each experiment was repeated three times.

\section{Additional file}

Additional file 1: Figure S1. The transcript levels of related chlorophyll metabolism in rice seedlings by pretreatment with an ethylene precursor under submergence conditions were detected by qRT-PCR (JPG 1103 kb)

\section{Abbreviations}

ACC:: 1-Aminocyclopropane-1-carboxylic acid; APX: Ascorbate peroxidase CAT: Catalase; $\mathrm{H}_{2} \mathrm{O}_{2}$ : Hydrogen peroxide; POX: Total peroxidase; ROS: Reactive oxygen species; RT-PCR: Reverse transcriptase-polymerase chain reaction;

SAGs: Senescence-associated genes; SOD: Superoxide dismutase

\section{Acknowledgements}

This manuscript was edited by Wallace Academic Editing.

\section{Funding}

This work was supported by a National Science Council grant (NSC 101-2311-B-005-001) to Chin-Ying Yang.

\section{Availability of data and materials}

All data generated or analysed during this study are included in this published article.

\section{Authors' contributions}

Y-C Huang and T-H Yeh conducted experiments and analyzed the data. Dr. C-Y Yang conceived, designed research, and wrote the manuscript.

All authors read and approved the final manuscript.

Ethics approval and consent to participate

Not applicable.

\section{Consent for publication}

Not applicable.

\section{Competing interests}

The authors declare that they have no competing interests.

\section{Publisher's Note}

Springer Nature remains neutral with regard to jurisdictional claims in published maps and institutional affiliations.

Received: 19 September 2018 Accepted: 2 April 2019

Published online: 11 April 2019

\section{References}

Adams DO, Yang SF (1979) Ethylene Biosynthesis - Identification of 1Aminocyclopropane-1-Carboxylic Acid as an Intermediate in the Conversion of Methionine to Ethylene. P Natl Acad Sci USA 76(1):170-174. https://doi. org/10.1073/pnas.76.1.170

Chen HJ, Qualls RG, Miller GC (2002) Adaptive responses of Lepidium latifolium to soil flooding: biomass allocation, adventitious rooting, aerenchyma formation and ethylene production. Environ Exp Bot 48(2):119-128. https:// doi.org/10.1016/S0098-8472(02)00018-7

El-Maarouf-Bouteau H, Sajjad Y, Bazin J, Langlade N, Cristescu SM, Balzergue S, Baudouin E, Bailly C (2015) Reactive oxygen species, abscisic acid and ethylene interact to regulate sunflower seed germination. Plant Cell Environ 38(2):364-374

Foyer CH, Noctor G (2005) Redox homeostasis and antioxidant signaling: a metabolic interface between stress perception and physiological responses. Plant Cell 17(7):1866-1875

Gautam P, Lal B, Tripathi R, Baig MJ, Shahid M, Maharana S, Bihari P, Nayak AK (2017) Impact of seedling age and nitrogen application on submergence 
tolerance of Sub1 and non-Sub1 cultivars of Rice (Oryza sativa L.). J Plant Growth Regul 36(3):629-642

Guo Y, Zhu CH, Gan LJ, Ng D, Xia K (2015) Ethylene is involved in the completesubmergence induced increase in root iron and manganese plaques in Oryza sativa. Plant Growth Regul 76(3):259-268

Hattori Y, Nagai K, Ashikari M (2011) Rice growth adapting to Deepwater. Curr Opin Plant Biol 14(1):100-105

Jackson MB, Hall KC (1987) Early stomatal closure in waterlogged pea-plants is mediated by abscisic-acid in the absence of foliar water deficits. Plant Cell Environ 10(2):121-130

Jackson MB, Ram PC (2003) Physiological and molecular basis of susceptibility and tolerance of rice plants to complete submergence. Ann Bot-London 91(2):227-241

Johnson PR, Ecker JR (1998) The ethylene gas signal transduction pathway: a molecular perspective. Annu Rev Genet 32:227-254

Kepczynski J, Kepczynska E (1997) Ethylene in seed dormancy and germination. Physiol Plantarum 101(4):720-726

Kumar V, Parvatam G, Ravishankar GA (2009) AgNO3 - a potential regulator of ethylene activity and plant growth modulator. Electron J Biotechnol 12(2)

Manzur ME, Grimoldi AA, Insausti P, Striker GG (2009) Escape from water or remain quiescent? Lotus tenuis changes its strategy depending on depth of submergence. Ann Bot-London 104(6):1163-1169

Matthews S, Khajeh-Hosseini M (2007) Length of the lag period of germination and metabolic repair explain vigour differences in seed lots of maize (Zea mays). Seed Sci Technol 35(1):200-212

Miro B, Ismail AM (2013) Tolerance of anaerobic conditions caused by flooding during germination and early growth in rice (Oryza sativa L.). Front Plant SC 4:269

Mittler R, Vanderauwera S, Suzuki N, Miller G, Tognetti VB, Vandepoele K, Gollery M, Shulaev V, Van Breusegem F (2011) ROS signaling: the new wave? Trends Plant Sci 16(6):300-309

Muhlenbock P, Plaszczyca M, Plaszczyca M, Mellerowicz E, Karpinski S (2007) Lysigenous aerenchyma formation in Arabidopsis is controlled by LESION SIMULATING DISEASE1. Plant Cell 19(11):3819-3830

Nishiuchi S, Yamauchi T, Takahashi H, Kotula L, Nakazono M (2012) Mechanisms for coping with submergence and waterlogging in rice. Rice 5

Ouaked F, Rozhon W, Lecourieux D, Hirt H (2003) A MAPK pathway mediates ethylene signaling in plants. EMBO J 22(6):1282-1288

Peng HP, Chan CS, Shih MC, Yang SF (2001) Signaling events in the hypoxic induction of alcohol dehydrogenase gene in Arabidopsis. Plant Physiol 126(2):742-749

Perez-Ramos IM, Maranon T (2009) Effects of waterlogging on seed germination of three Mediterranean oak species: ecological implications. Acta Oecol 35(3): $422-428$

Petruzzelli L, Coraggio I, Leubner-Metzger G (2000) Ethylene promotes ethylene biosynthesis during pea seed germination by positive feedback regulation of 1-aminocyclo-propane-1-carboxylic acid oxidase. Planta 211(1):144-149

Pruzinska A, Anders I, Aubry S, Schenk N, Tapernoux-Luthi E, Muller T, Krautler B, Hortensteiner S (2007) In vivo participation of red chlorophyll catabolite reductase in chlorophyll breakdown. Plant Cell 19(1):369-387

Rajhi I, Yamauchi T, Takahashi H, Nishiuchi S, Shiono K, Watanabe R, Mliki A, Nagamura Y, Tsutsumi N, Nishizawa NK, Nakazono M (2011) Identification of genes expressed in maize root cortical cells during lysigenous aerenchyma formation using laser microdissection and microarray analyses. New Phytol 190(2):351-368

Reddy MD, Mittra BN (1985) Effects of complete plant submergence on vegetative growth, grain-yield and some biochemical-changes in Rice plants. Plant Soil 87(3):365-374

Rzewuski G, Sauter M (2008) Ethylene biosynthesis and signaling in rice. Plant Sci 175(1-2):32-42. https://doi.org/10.1016/j.plantsci.2008.01.012

Sarlistyaningsih L, Sivasithamparam K, Setter TL (1995) Influence of waterlogging on germination and survival of Lupin seeds (Lupinus-Angustifolius L cv Gungurru) coated with calcium peroxide and streptomycin. Aust J Exp Agr 35(4):537-541

Steffens B (2014) The role of ethylene and ROS in salinity, heavy metal, and flooding responses in rice. Front Plant Sci 5

Strader LC, Beisner ER, Bartel B (2009) Silver ions increase auxin efflux independently of effects on ethylene response. Plant Cell 21(11):3585-3590

Teige M, Scheikl E, Eulgem T, Doczi F, Ichimura K, Shinozaki K, Dangl JL, Hirt H (2004) The MKK2 pathway mediates cold and salt stress signaling in Arabidopsis. Mol Cell 15(1):141-152
Wu YS, Yang CY (2016) Physiological responses and expression profile of NADPH oxidase in Rice (Oryza Sativa) seedlings under different levels of submergence. Rice 9

Xia XJ, Zhou YH, Shi K, Zhou J, Foyer CH, Yu JQ (2015) Interplay between reactive oxygen species and hormones in the control of plant development and stress tolerance. J Exp Bot 66(10):2839-2856

Yamada Y, Furusawa S, Nagasaka S, Shimomura K, Yamaguchi S, Umehara M (2014) Strigolactone signaling regulates rice leaf senescence in response to a phosphate deficiency. Planta 240(2):399-408

Yang CY, Hong CP (2015) The NADPH oxidase Rboh D is involved in primary hypoxia signalling and modulates expression of hypoxia-inducible genes under hypoxic stress. Environ Exp Bot 115:63-72

Yang CY, Hsu FC, Li JP, Wang NN, Shih MC (2011) The AP2/ERF transcription factor AtERF73/HRE1 modulates ethylene responses during hypoxia in Arabidopsis. Plant Physiol 156(1):202-212

Yang SF (1985) Biosynthesis and action of ethylene. Hortscience 20(1):41-45

Yang SY, Wu YS, Chen CT, Lai MH, Yen HM, Yang CY (2017) Physiological and molecular responses of seedlings of an upland rice ('Tung Lu $3^{\prime}$ ) to total submergence compared to those of a submergence-tolerant lowland rice ('FR13A'). Rice 10(1):42

Yu YW, Yang DX, Zhou SR, Gu JT, Wang FR, Dong JG, Huang RF (2017) The ethylene response factor OsERF109 negatively affects ethylene biosynthesis and drought tolerance in rice. Protoplasma 254(1):401-408

\section{Submit your manuscript to a SpringerOpen ${ }^{\circ}$ journal and benefit from:}

- Convenient online submission

- Rigorous peer review

- Open access: articles freely available online

- High visibility within the field

- Retaining the copyright to your article

Submit your next manuscript at $\boldsymbol{\nabla}$ springeropen.com 